\title{
Beneficial effects of grape seed proanthocyanidin extract on arterial remodeling in spontaneously hypertensive rats via protecting against oxidative stress
}

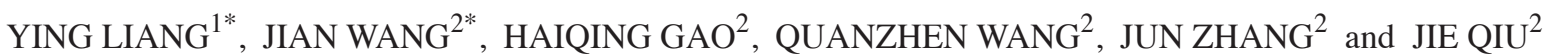 \\ ${ }^{1}$ Department of Geriatric Cardiology, Qianfoshan Hospital of Shandong University, Jinan, Shandong 250013; \\ ${ }^{2}$ Department of Geriatric Cardiology, Qilu Hospital of Shandong University, Jinan, Shandong 250012, P.R. China
}

Received August 2, 2015; Accepted August 1, 2016

DOI: $10.3892 / \mathrm{mmr} .2016 .5699$

\begin{abstract}
Arterial remodeling is a pathogenic occurrence during hypertension and, in turn, is closely associated with the development and complications of hypertension. Grape seed proanthocyanidin extract (GSPE) has been reported to exhibit a protective effect on cardiovascular disease, however its effect on arterial remodeling remains to be fully elucidated. In the present study, the effects of GSPE on arterial remodeling were analyzed by treating spontaneously hypertensive rats (SHRs) with GSPE (250 mg/kg·day). Arterial remodeling was quantified through morphological methods; thoracic aortas were stained with hematoxylin-eosin or sirius red-victoria blue. The arterial ultrastructure was imaged using transmission electron microscopy. The content of nitric oxide (NO) and endothelin-1 (ET-1) were examined to determine endothelial function. Oxidative stress was assessed by malondialdehyde (MDA) levels and the activities of the antioxidant enzymes superoxide dismutase (SOD) and catalase (CAT). Administration of GSPE markedly alleviated hypertension-induced arterial remodeling, which was not associated with blood pressure control. ET-1 production was reduced, while NO production was increased in the GSPE group, which exhibited improved endothelial function. In addition, treatment with GSPE significantly ameliorated oxidative stress by improving SOD and CAT activities and reducing MDA formation. In conclusion, GSPE may attenuate hypertension-induced arterial remodeling by repressing oxidative stress and is recommended as a potential anti-arterial remodeling agent for patients with hypertensive vascular diseases.
\end{abstract}

Correspondence to: Professor Jie Qiu, Department of Geriatric Cardiology, Qilu Hospital of Shandong University, 107 Wenhua Xi Road, Jinan, Shandong 250012, P.R. China

E-mail:drqiujie@163.com

\section{${ }^{*}$ Contributed equally}

Key words: grape seed proanthocyanidin extract, arterial remodeling, oxidative stress, hypertension

\section{Introduction}

Hypertension is a major health problem and predominant risk factor for the occurrence of numerous diseases, including heart failure, myocardial infarction, stroke and peripheral arterial disease $(1,2)$. Hypertension is capable of promoting arterial remodeling, which is an adaptive process that occurs in response to long-term alterations in the hemodynamic condition of hypertension, predominantly including vessel wall thickening and histological abnormalities, hemal wall/lumen ratio increases and endothelial dysfunction (3). The process of arterial remodeling is fundamental to numerous vascular diseases that remain challenging to effectively treat. Thus, therapeutic strategies directed at influencing the remodeling response may have clinical importance.

It is evident that oxidative stress is associated with the pathogenesis of numerous diseases, including vascular injury (4). In addition, it has been reported that oxidative stress is present in the arterial remodeling of hypertension. Reactive oxygen species (ROS) at moderate concentrations act as signaling molecules and second messengers, which serve an important role in maintaining the structure and function of the vascular integrity $(5,6)$. In contrast to these regulatory functions under physiological conditions, excessive or sustained ROS production, when exceeding the available antioxidant defense systems, leads to oxidative stress. Oxidative stress damages the endothelium and impairs endothelium-dependent vasodilatation, consequently resulting in endothelial dysfunction, and promoting the proliferation of vascular smooth muscle cells in addition to collagen deposition, which cause thickening of the tunica media and narrowing of the vascular lumen (7). This results in the occurrence and the development of hypertension $(8,9)$. These observations demonstrate that oxidative stress is involved in the occurrence and development of arterial remodeling in hypertension, and suggest that arterial remodeling may be alleviated by its inhibition.

Grape seed proanthocyanidin extract (GSPE) is a combination of biologically active polyphenolic flavonoids, and contains oligomeric proanthocyanidins, which have been demonstrated to exhibit a spectrum of biological, pharmacological, therapeutic and chemoprotective properties against oxygen-free radicals and oxidative stress (10). This range of biochemical and cellular functions suggests potential for the prevention and treatment of 
a variety of human disorders caused by oxidative stress. Due to the fact that oxidative damage has been associated with the arterial remodeling in hypertension, the curernt study aimed to evaluate the effect of GSPE on arterial remodeling, which has not, to the best of our knowledge been previously investigated.

\section{Materials and methods}

Animal preparation. A total of 2020 -week-old male spontaneously hypertensive rats (SHRs) weighing 302.25 $\pm 7.29 \mathrm{~g}$ and 10 20-week-old Wistar-Kyoto rats (WKYs) weighing 298.25 $\pm 6.40 \mathrm{~g}$ were purchased from Vital River Laboratory Animal Co., Ltd. (Beijing, China). The rats were kept in cages at $22 \pm 2^{\circ} \mathrm{C}$ and $50-55 \%$ humidity with 12 -h light/dark cycles and had access to standard rat feed and water ad libitum. The rats were randomly assigned to three groups $(n=10$ per group): WKY-C (WKY control rats treated with $1 \mathrm{ml} 0.9 \%$ nitric sodium orally), SHR-C (SHR control rats treated with $1 \mathrm{ml} 0.9 \%$ nitric sodium orally) and SHR-T (SHRs treated with GSPE at a dosage of $250 \mathrm{mg} / \mathrm{kg} \cdot$ day). In previous studies, rats treated with GSPE at a dosage of $250 \mathrm{mg} / \mathrm{kg}$.day exhibited a more significant biological effect without pharmacological toxicity $(11,12)$. The animals were subjected to drug administration as described above by oral gavage for 22 weeks. All experiments were approved and performed in accordance with the National Institute of Health Guide for the Care and Use of Laboratory Animals (13), with approval from the Institutional Animal Care and Use Committee of Qilu Hospital, Shandong University (Jinan, China).

Chemicals. GSPEs (containing 56\% dimeric proanthocyanidins, $12 \%$ trimeric proanthocyanidins, $6.6 \%$ tetrameric proanthocyanidins and small amounts of monomeric and high-molecular-weight oligomeric proanthocyanidins and flavanols) were provided by Tianjin Jianfeng Natural Product R\&D Co., Ltd. (Tianjin, China). The components of GSPE were analyzed using high-performance liquid chromatography with gas chromatography-mass spectrometry detection.

Systolic blood pressure(SBP) measurement. SBP was measured in conscious animals prior to the start of treatment and weekly during treatment. SBP was determined using the tail-cuff method (BP-2006A; Softron Beijing Incorporated, Beijing, China). Prior to measurement, animals were placed in a heated chamber at an ambient temperature of $30-34^{\circ} \mathrm{C}$ for $15 \mathrm{~min}$ and were conditioned to numerous cuff inflation-deflation cycles by a trained operator. Three consecutive SBP readings were collected and averaged to obtain the exact SBP for presentation.

Tissue collection. At the end of week 22, animals in all groups were sacrificed by decapitation under $3 \%$ sodium pentobarbital. Thoracic aortas were removed completely and rapidly. Sections of the thoracic aorta $(4 \mu \mathrm{m})$ were fixed in buffered $10 \%$ formalin for hematoxylin-eosin or sirius red-victoria blue staining, while others were frozen in liquid nitrogen and stored at $-80^{\circ} \mathrm{C}$ for the biochemical assays.

Histopathological evaluation. Specimens of the middle part of the thoracic aorta were fixed for $24 \mathrm{~h}$ in $10 \%$ formalin, routinely processed in paraffin and cut into $4-\mu \mathrm{m}$-thick slices for staining with hematoxylin-eosin or sirius red-victoria blue. Briefly, the paraffin sections were deparaffinized and rehydrated in distilled water. Following washing with $70 \%$ ethanol for $2 \mathrm{~min}$, the sections were stained in victoria blue solution for $12 \mathrm{~h}$ at $37^{\circ} \mathrm{C}$. The sections were then briefly washed with $95 \%$ ethanol for several seconds and with distilled water for $2 \mathrm{~min}$ prior to staining with $1 \%$ picrosirius red F3BA (Sigma-Aldrich; Merck Millipore, Darmstadt, Germany) for $1 \mathrm{~h}$. The sections were then washed in running tap water for $10 \mathrm{~min}$ prior to dehydration, clearing and mounting. Victoria blue- and picrosirius red-stained sections were visualized by bright field and polarized light under an Olympus BX53 microscope (Olympus Corporation, Tokyo, Japan) and measured using Image-Pro Plus software, version 5.0 (Media Cybernetics, Inc., Rockville, MD) to obtain the percentage of collagen per media area and the collagen-elastic ratio. The wall thickness (WT), inner diameter (ID) and aorta radius (AR) of the thoracic aorta were determined using an Olympus DP71 camera (Olympus Corporation), and then the outer diameter (OD), vascular cross-sectional area (VCSA), wall cross-sectional area (WSCA), luminal cross-sectional area (LCSA), wall-lumen ratio (wall thickness:inner diameter) and WSCA/LCSA were calculated.

Ultrastructural examination. A portion of the thoracic aorta was fixed in 3\% glutaraldehyde (Nanjing Chemical Reagent Co., Ltd., Nanjing, China). Ultrathin sections cut from the embedded blocks were stained with uranyl acetate and lead citrate and then observed with an H-800 transmission electron microscope (Hitachi, Ltd., Tokyo, Japan).

Pulse wave velocity $(P W V)$. Two polyethylene cannulas were inserted into the aorta via the left carotid and the left femoral arteries for central and peripheral blood pressure measurements, respectively. The aorta cannulas were connected to a pressure recording system (MP100CE) and with AcqKnowledge software, version 3.7.3 (BioPAC Systems, Inc., Goleta, CA, USA). PWV (cm/s) was calculated as the distance between the central and peripheral cannula tips divided by the transit time. The distance between the two central and peripheral cannula tips was measured in situ subsequent to postmortem fixation by sticking a damp cotton thread onto the aorta. Transit times between the two central and peripheral pressure signals were measured online for each $5 \mathrm{sec}$ period by peak detectors of the AcqKnowledge software, which systematically shifted the peripheral pressure waveform in time with respect to the central pressure waveform and determined the value of the time.

Enzyme-linked immunosorbent assay. The aorta tissue was sectioned as small as possible that were homogenized 1:9 (w:v) in $0.9 \%$ saline. The homogenates were then centrifuged at $1,500 \mathrm{xg}$ for $5 \mathrm{~min}$ at $4^{\circ} \mathrm{C}$, and the supernatant was used to determine the content of nitric oxide (NO) and endothelin-1 (ET-1) and the levels of superoxide dismutase (SOD), catalase (CAT) and malondialdehyde (MDA). The quantity of NO and ET-1 in the aorta tissues was measured according to manufacturer's instructions using the NO Assay kit and the ET-1 Assay kit, respectively (Nanjing Jiancheng Bioengineering Institute, Nanjing, China). The levels of SOD, CAT and MDA in aorta tissue were performed according to manufacturer's instructions using the SOD Assay kit, the CAT Assay kit and the 
Table I. Effect of grape seed proanthocyanidin extract on the parameters of arterial remodeling.

\begin{tabular}{lcrr}
\hline & & Groups & \\
\cline { 2 - 4 } Parameter & WKY-C & SHR-C & SHR-T \\
\hline WT $(\mu \mathrm{m})$ & $109.99 \pm 17.14$ & $234.69 \pm 51.76^{\mathrm{b}}$ & $123.44 \pm 21.52^{\mathrm{d}}$ \\
ID $(\mu \mathrm{m})$ & $1939.72 \pm 188.25$ & $2286.03 \pm 37.18^{\mathrm{a}}$ & $2146.63 \pm 121.73$ \\
Wall-lumen ratio $(\%)$ & $5.76 \pm 1.40$ & $10.26 \pm 2.27^{\mathrm{a}}$ & $5.74 \pm 0.77^{\mathrm{c}}$ \\
OD $(\mu \mathrm{m})$ & $2551.94 \pm 168.60$ & $2863.10 \pm 94.76^{\mathrm{b}}$ & $2457.40 \pm 160.16^{\mathrm{c}}$ \\
AR $(\mu \mathrm{m})$ & $1079.85 \pm 77.17$ & $1377.71 \pm 56.68^{\mathrm{b}}$ & $1196.75 \pm 78.02^{\mathrm{c}}$ \\
VCSA $\left(\mu \mathrm{m}^{2}\right)$ & $3995782.31 \pm 600797.63$ & $6439627.69 \pm 423165.96^{\mathrm{b}}$ & $4753893.19 \pm 624694.74^{\mathrm{c}}$ \\
WCSA $\left(\mu \mathrm{m}^{2}\right)$ & $1023674.09 \pm 31811.67$ & $2336546.56 \pm 374561.53^{\mathrm{b}}$ & $1128830.56 \pm 241649.31^{\mathrm{d}}$ \\
LCSA $\left(\mu \mathrm{m}^{2}\right)$ & $2972108.22 \pm 580826.92$ & $4103081.13 \pm 133875.61^{\mathrm{a}}$ & $3625062.64 \pm 410015.65$ \\
WCSA $/ \mathrm{LCSA}(\%)$ & $35.21 \pm 5.86$ & $56.94 \pm 9.05^{\mathrm{b}}$ & $31.01 \pm 4.04^{\mathrm{d}}$ \\
PWV $(\mathrm{cm} / \mathrm{s})$ & $2492.10 \pm 789.70$ & $6681.09 \pm 2154.93$ & $4283.10 \pm 946.05$ \\
\hline
\end{tabular}

Values are presented as the means \pm standard deviation for six rats. ${ }^{a} \mathrm{P}<0.05,{ }^{b} \mathrm{P}<0.01$ vs. WKY-C group; ${ }^{\mathrm{c}} \mathrm{P}<0.05$, ${ }^{\mathrm{d}} \mathrm{P}<0.01 \mathrm{vs}$. SHR-C group. WKY-C, Wistar-Kyoto control rats; SHR-C, spontaneously hypertensive control rats; WT, wall thickness; ID, inner diameter; wall-lumen ratio, ratio of WT to ID; OD, outer diameter; AR, aorta radius; VCSA, vascular cross-sectional area; WCSA, wall cross-sectional area; LCSA, luminal cross-sectional area; PWV, pulse wave velocity.

A

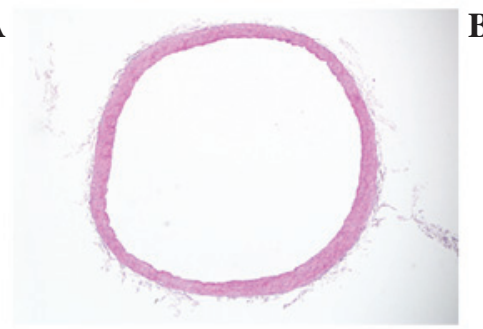

D

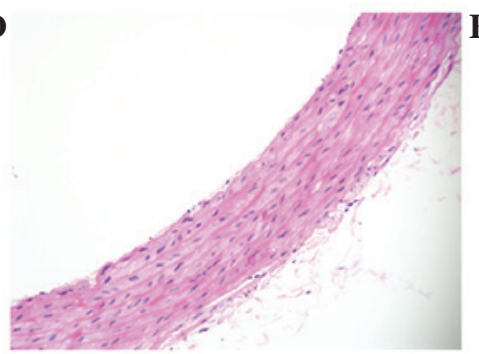

B

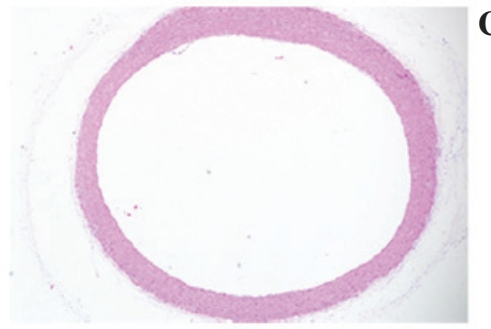

$\mathbf{E}$

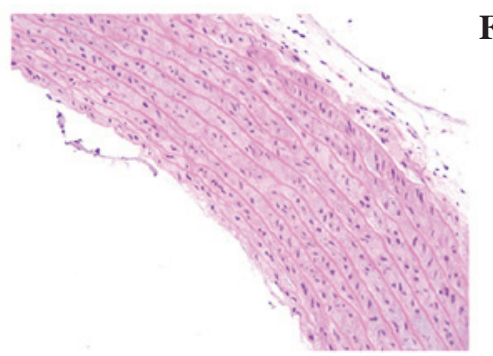

C

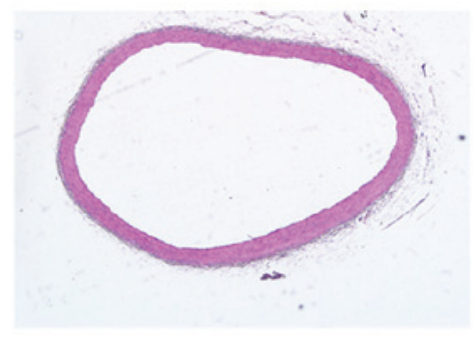

$\mathbf{F}$

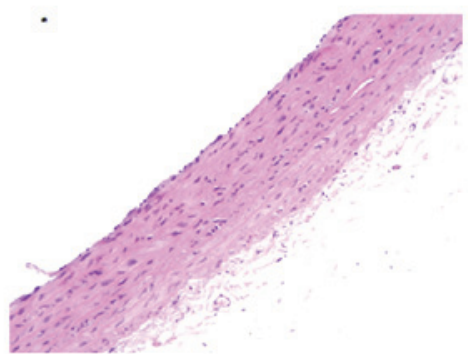

Figure 1. Effect of GSPE on histological sections of thoracic aorta stained with hematoxylin-eosin in the three groups (magnification: A-C, x40; D-F, x400). (A and D) WKY-C group; (B and E) SHR-C group; (C and F) SHR-T group. GSPE, grape seed proanthocyanidin extract; WKY-C, Wistar-Kyoto rats treated with $1 \mathrm{ml} \mathrm{0.9 \%} \mathrm{nitric} \mathrm{sodium} \mathrm{orally;} \mathrm{SHR-C,} \mathrm{spontaneously} \mathrm{hypertensive} \mathrm{rats} \mathrm{treated} \mathrm{with} 1 \mathrm{ml} 0.9 \%$ nitric sodium orally; SHR-T, SHRs treated with GSPEs at a dosage of $250 \mathrm{mg} / \mathrm{kg} \cdot$ day.

Microscale MDA Assay kit, respectively (Nanjing Jiancheng Bioengineering Institute).

Statistical analysis. Statistical analysis was performed by one-way analysis of variance using SPSS software, version 19.0 (SPSS, Inc., Chicago, IL, USA). The results are expressed as the mean \pm standard deviation. $\mathrm{P}<0.05$ was considered to indicate a statistically significant difference.

\section{Results}

GSPE ameliorates alterations in arterial remodeling. As presented in Table I, wall thickness, OD, AR, VCSA, WCSA, wall-lumen ratio, WCSA/LCSA and PWV were significantly increased in the SHR-C group compared with the WKY-C group. Following administration of GSPE (at a dose of $250 \mathrm{mg} / \mathrm{kg} \cdot$ day), the above parameters were reversed, indicating that GSPE reversed arterial remodeling. To further investigate the effect of GSPE on arterial remodeling, thoracic aortas were stained with hematoxylin-eosin or sirius red-victoria blue.

Hematoxylin and eosin staining indicated that the arrangement of the elastic fibers in the aortas in the WKY-C group rats was normal and that there was no collagen hyperplasia in the vessel wall, while the aortic wall in the SHR-C group rats was thickened, with hyperplastic collagen fibers in the media 
A
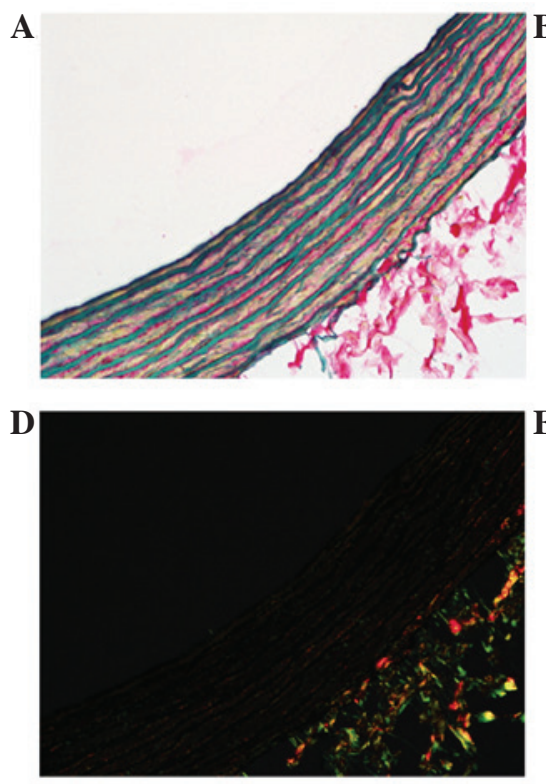
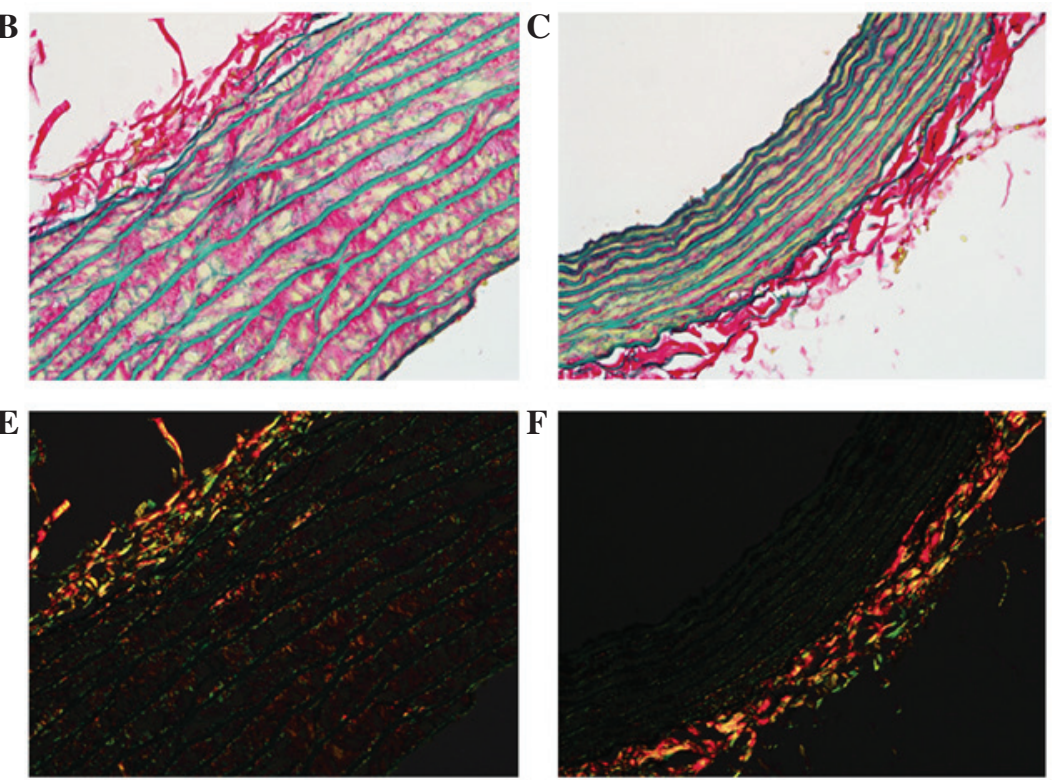

Figure 2. Effect of GSPE on aortic fibrosis. (A-C) Representative images of bright fields. (D-F) Representative histological images of polarized light (A and D) WKY-C group; (B and E) SHR-C group; (C and F) SHR-T group. GSPE, grape seed proanthocyanidin extract; WKY-C, Wistar-Kyoto rats treated with $1 \mathrm{ml} 0.9 \%$ nitric sodium orally; SHR-C, spontaneously hypertensive rats treated with $1 \mathrm{ml} 0.9 \%$ nitric sodium orally; SHR-T, SHRs treated with GSPEs at a dosage of $250 \mathrm{mg} / \mathrm{kg} \cdot$ day.
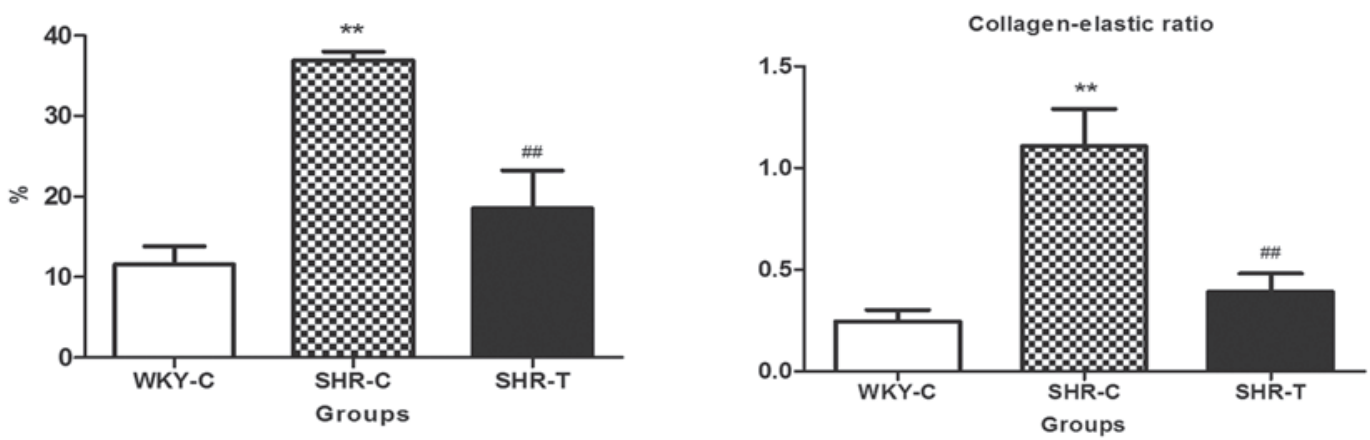

Figure 3. Quantitative assessment of $\%$ area of collagen fibers and collagen-elastic ratio in the three groups. Data are presented as the mean \pm standard deviation; $\mathrm{n}=10 .{ }^{* *} \mathrm{P}<0.01$ vs. the WKY-C group; ${ }^{\# \#} \mathrm{P}<0.01$ vs. the SHR-C group. WKY-C, Wistar-Kyoto rats treated with 1 ml $0.9 \%$ nitric sodium orally; SHR-C spontaneously hypertensive rats treated with $1 \mathrm{ml} \mathrm{0.9 \%} \mathrm{nitric} \mathrm{sodium} \mathrm{orally;} \mathrm{GSPE,} \mathrm{grape} \mathrm{seed} \mathrm{proanthocyanidin} \mathrm{extract;} \mathrm{SHR-T,} \mathrm{SHRs} \mathrm{treated} \mathrm{with} \mathrm{GSPEs}$ at a dosage of $250 \mathrm{mg} / \mathrm{kg} \cdot \mathrm{day}$.

and with reduced, disordered and even ruptured elastic fibers. However, the aortic elastic fibers in the SHR-T group remained ordered (Fig. 1).

Sirius red-victoria blue staining was observed in the histological images (Fig. 2), where red fibers represent collagen and blue fibers show elastin in the bright fields, and under polarized light, the collagen I fibers presented orange-red, whereas the thinner collagen III fibers appeared yellow-green (14). Previous studies demonstrated that certain conditions, such as hypertension and atherosclerosis, stimulate vascular smooth muscle cells (VSMCs) to produce extra collagen in the vessel wall $(15,16)$ and that the arterial collagen content increases progressively when blood pressure rises $(17,18)$.

The present study supports this theory by demonstrating a marked increase in total collagen content per media area of the aortic segment for the SHRs compared with the WKY rats. In the SHR-C group, the aortic fibration was promoted by increasing collagen deposition in the wall, particularly collagen type I, while it was lower in the SHR-T group when compared with the SHR-C group. In addition, the ratio of collagen fibers and elastic fibers was reduced more in the SHR-T group than in the SHR-C group. Collagen content and the collagen-elastic ratio were lower in the SHR-T group than in the SHR-C tissues, indicating that GSPE reduced collagen deposition (Fig. 3).

GSPE preserves ultrastructural alterations of the thoracic aorta. In the SHR-C group, the repeatedly repaired basement membrane had numerous holes, and the internal elastic lamina was split, with abundant extracellular matrix content intertwined with the basement membrane. In addition, fibroblast-like cells were present in the subendothelium, and the apoptosis of endothelial cells was observed in the SHR-C group, whereas normal ultrastructure was observed in the aortic tissues of the WKY-C group. The administration of 


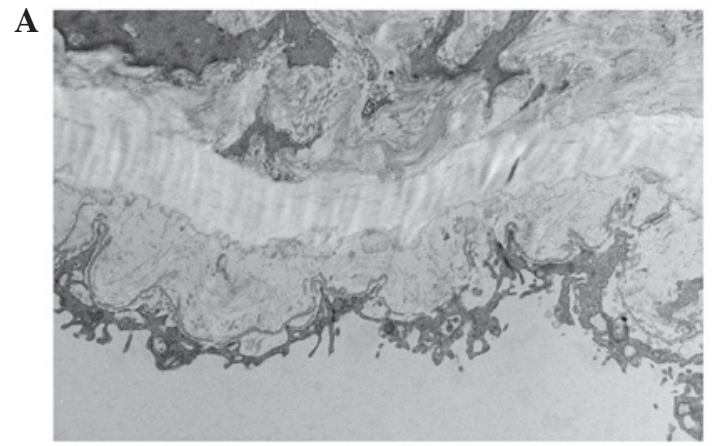

$\mathbf{B}$
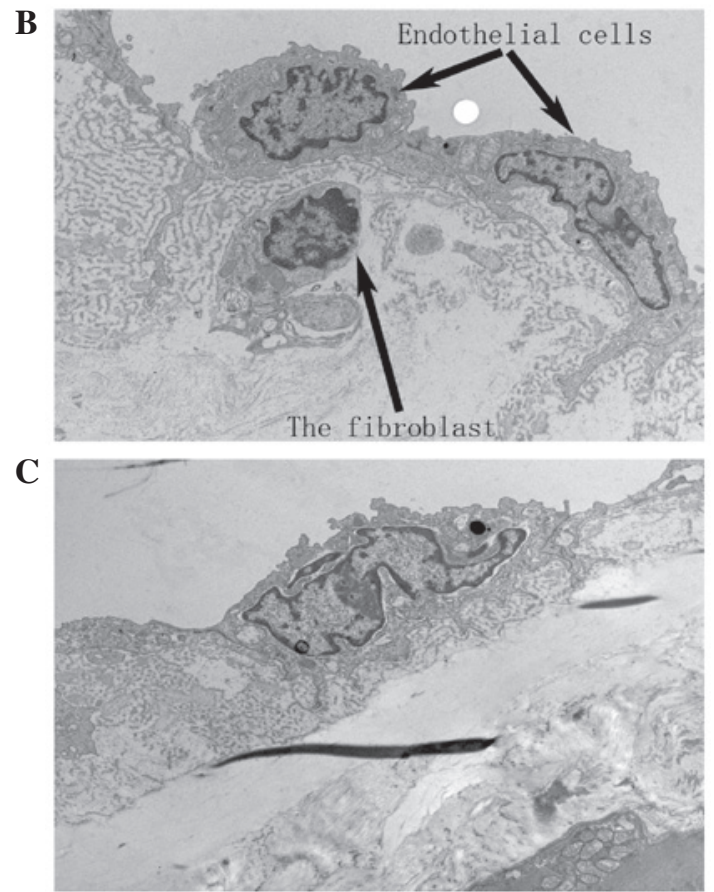

Figure 4. Effect of GSPEs on ultrastructural alterations in the thoracic aorta in the three groups (magnification, x15,000). (A) WKY-C group; (B) SHR-C group; (C) SHR-T group. GSPE, grape seed proanthocyanidin extract WKY-C, Wistar-Kyoto rats treated with $1 \mathrm{ml} 0.9 \%$ nitric sodium orally; SHR-C, spontaneously hypertensive rats treated with $1 \mathrm{ml} 0.9 \%$ nitric sodium orally; SHR-T, SHRs treated with GSPEs at a dosage of $250 \mathrm{mg} / \mathrm{kg} \cdot$ day.

GSPE resulted in improvements to the preservation of the fine structure of the basement membrane and internal elastic lamina and a reduction in the number of inserted fibroblast-like cells in the subendothelium (Fig. 4).

GSPE significantly improves endothelial function. The content of NO in the thoracic aorta in the SHR-C group reduced significantly, while GSPE treatment increased NO production when comparing the SHR-T group with the SHR-C group. However, ET-1 expression was significantly increased in the SHR-C group when compared with that of the WKY-C group, which was capable of being reversed by GSPE treatment, indicating that GSPE significantly improved endothelial function (Fig. 5).

GSPE acts via the reduction of oxidative stress. During the 22 weeks of treatment, SBP was similarly increased in both the SHR-C and the SHR-T groups, indicating that GSPE had no effect on blood pressure (Fig. 6). As presented in Fig. 7, compared with the WKY-C group, MDA expression was
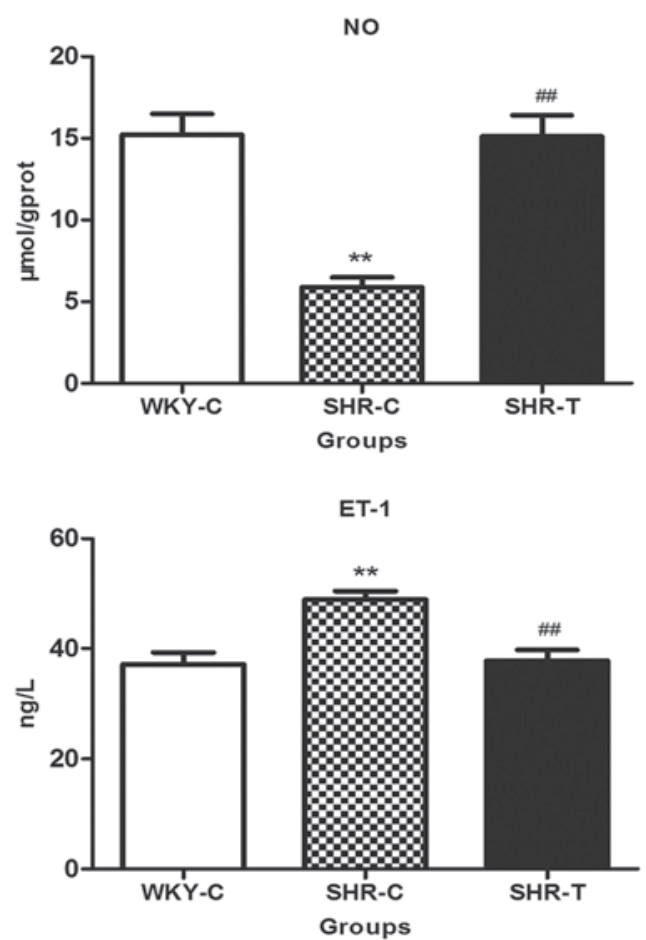

Figure 5. Effect of GSPE on the expression of NO and ET-1. The content of NO and ET-1 in the thoracic aorta from the three groups. NO, nitric oxide; ET-1, endothelin-1; groups as described in Fig. 1. Data are presented as the mean \pm standard deviation; $\mathrm{n}=10$. ${ }^{* *} \mathrm{P}<0.01$ vs. the WKY-C group; ${ }^{\# \#} \mathrm{P}<0.01$ vs. the SHR-C group. GSPE, grape seed proanthocyanidin extract; NO, nitric oxide; ET-1, endothelin-1; WKY-C, Wistar-Kyoto rats treated with $1 \mathrm{ml} 0.9 \%$ nitric sodium orally; SHR-C, spontaneously hypertensive rats treated with $1 \mathrm{ml} 0.9 \%$ nitric sodium orally; SHR-T, SHRs treated with GSPEs at a dosage of $250 \mathrm{mg} / \mathrm{kg} \cdot$ day.

Systolic blood pressure

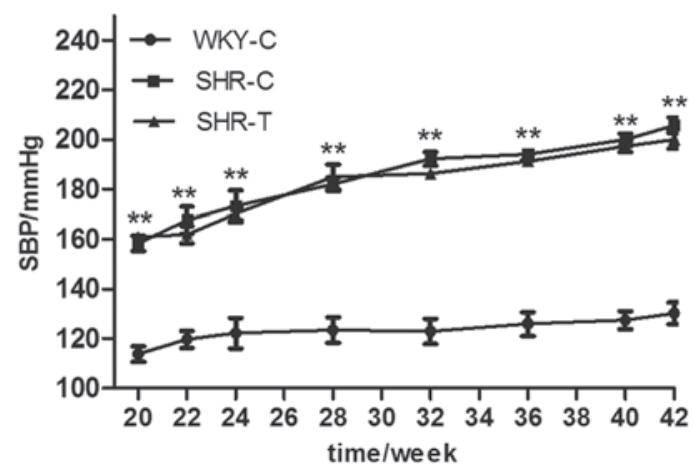

Figure 6. Effect of GSPE on SBP. SBP ( $\mathrm{mmHg}$ ) was measured across the three groups at baseline and at weekly intervals. Data are presented as the mean \pm standard deviation; $n=10 .{ }^{* *} \mathrm{P}<0.01$ vs. the WKY-C group. GSPE, grape seed proanthocyanidin extract; SBP, systolic blood pressure; WKY-C, Wistar-Kyoto rats treated with $1 \mathrm{ml} 0.9 \%$ nitric sodium orally; SHR-C, spontaneously hypertensive rats treated with $1 \mathrm{ml} 0.9 \%$ nitric sodium orally; SHR-T, SHRs treated with GSPEs at a dosage of $250 \mathrm{mg} / \mathrm{kg}$.day.

greater in the aorta tissue of the SHRs, while SOD and CAT activities were reduced, which was accompanied by arterial remodeling in SHRs, as demonstrated by a significant increase in wall thickness, wall-lumen ratio (Table I) and increased collagen fibers (Fig. 3). All of these parameters were reversed in SHRs chronically supplemented with GSPE. 
SOD

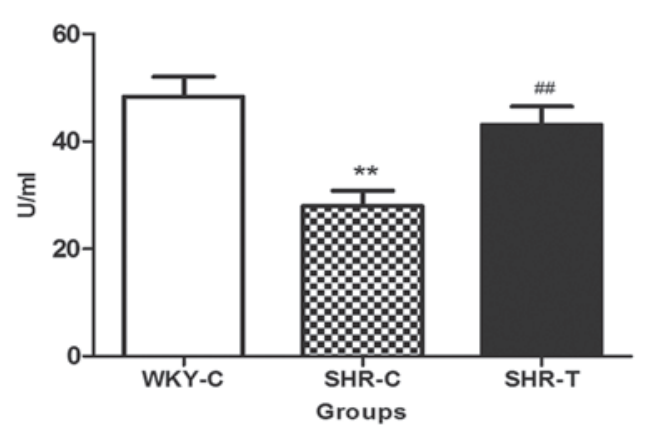

CAT

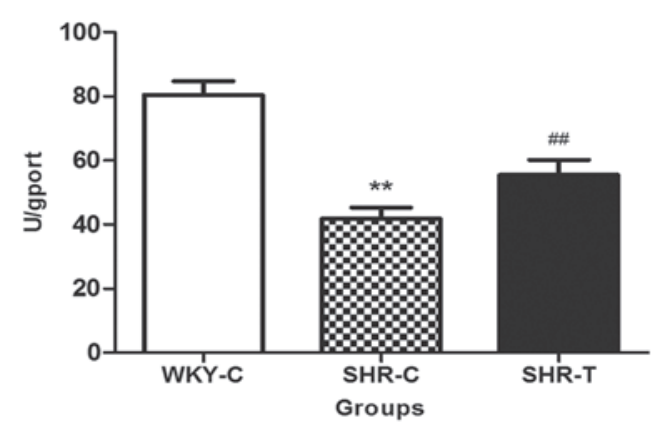

MDA

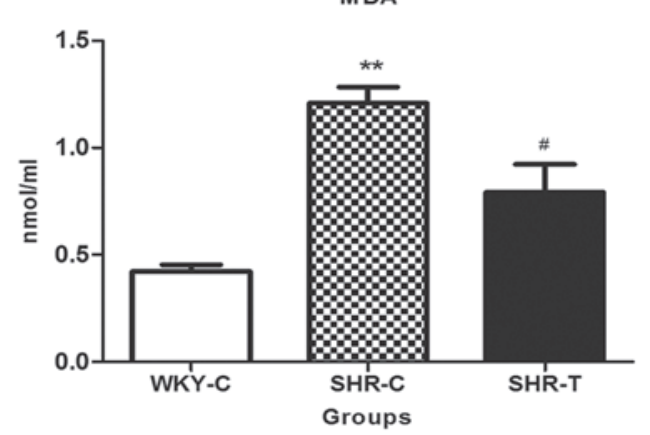

Figure 7. Effect of GSPE on levels of SOD, CAT and MDA. Data are presented as the mean \pm standard deviation; $n=10$. ${ }^{* *} \mathrm{P}<0.01$ vs. the WKY-C group; ${ }^{\# \#} \mathrm{P}<0.01$ vs. the SHR-C group. GSPE, grape seed proanthocyanidin extract; SOD, superoxide dismutase; CAT, catalase; MDA, malondialdehyde; WKY-C, Wistar-Kyoto rats treated with $1 \mathrm{ml} 0.9 \%$ nitric sodium orally; SHR-C, spontaneously hypertensive rats treated with $1 \mathrm{ml} 0.9 \%$ nitric sodium orally; SHR-T, SHRs treated with GSPEs at a dosage of $250 \mathrm{mg} / \mathrm{kg} \cdot$ day.

\section{Discussion}

Arterial remodeling is an active process of structural alteration that involves the vessel wall thickening and histological abnormalities, hemal wall/lumen ratio increases and finally endothelial dysfunction. Morphological observations from the current study indicated that a marked increase in wall thickness, OD, AR, VCSA, WCSA, wall-lumen ratio and WCSA/LCSA was observed in the SHR-C groups. Previous studies suggested that arterial remodeling leads to increased wall thickness and wall-lumen ratios, which appears to be due to degradation and reorganization of the extracellular matrix scaffold, in addition to hypertrophy and/or hyperplasia of the VSMCs $(11,16)$. Consistent with these results, collagen deposition was observed in the aortas of the SHR group. Pulse wave velocity (PWV), which is defined as the propagation speed of the pressure or flow wave front traveling along the aorta, has been regarded as a predictor for cardiovascular events and mortality $(19,20)$. The increased PWV reflected arterial stiffness as a result of structural alterations in the arterial wall (21). The structure of vessels is altered when an increase in blood pressure causes an augmentation of vascular tension in hypertension. These effects, in turn, lead to an increase in aortic wall stiffness and a quickening of PWV in hypertension. In the current study, SHRs exhibited a significantly increased PWV.

A previous study demonstrated that the imbalance of endothelium-derived factors may elevate vasomotor tone, promote VSMC proliferation and induce arterial remodeling (11). NO is the key endothelium-derived relaxing factor that serves an important role in the regulation of vascular function, and it appears that the abnormalities in the production or actions of NO lead to endothelial dysfunction and abnormal arterial remodeling (22). ET-1 is the dominant vasoconstrictive factor. A previous study identified that aortic ET-1 content is significantly increased in deoxycorticosterone acetate-salt hypertensive rats compared with that of age-matched control rats (23). Studies investigating clinical and experimental hypertension observed an imbalance between NO and ET-1 $(24,25)$. In the current study, an increase in ET-1 and a reduction in NO were observed. The present study confirms that SHRs have endothelial dysfunction, which may contribute to arterial remodeling.

As identified in the current study, the process of arterial remodeling leads to increased wall thickness, VCSA, WCSA, AR, wall-lumen ratio, WCSA/LCSA and PWV, which appear to be due to arterial wall hyperplasia and hypertrophy, and an imbalance in NO and ET-1 leading to endothelial dysfunction. However, these alterations were reversed by GSPE. A previous study reported that phenolic compounds prevent target organ damage in hypertensive rats (26). GSPE, a phenolic compound, has been reported to protect against oxidative injury during doxorubicin-induced/cyclosporine-induced cardiac injury and ischemia/reperfusion in the rat heart (27-29). In a controlled registry study involving 119 healthy, pre- and mildly hypertensive subjects (30), GSPE significantly reduced blood pressure, however, in the present study, its effect could not be observed in the SHRs. In the current study, the starting time of treatment, age and weight of the SHRs, which may serve an important role in the treatment of high blood pressure, were different from previous studies $(11,12)$. It is suggested that this explains the discrepancies between the results obtained in the current study and those of previous studies. These results indicated that the 
vascular remodeling was improved independent of reducing blood pressure, however was associated with the direct effects of GSPE. Therefore, it is hypothesized that significantly reduced wall thickness, OD, AR, VCSA, WCSA, wall-lumen ratio, WCSA/LCSA, PWV and ET-1, and increased NO in the SHR-T group may be due to the antioxidant activity of GSPE.

Oxidative stress occurs when there is an excessive or sustained ROS production that exceeds the available antioxidant defense systems. ROS are highly reactive and unstable by nature; hence, they can damage various cellular components, including lipid membranes. Lipid peroxides are derived from polyunsaturated fatty acid (PUFA) oxidation and are capable of initiating lipid peroxidation via a free radical chain reaction. MDA is a major end-product of PUFA peroxidation and is often used as an indicator of cell injury. An increase in the production of MDA may be due to the formation of reactive oxidants. GSPE significantly inhibited oxidative stress, as indicated by the reduced level of MDA in SHRs treated with GSPE. The increase in MDA in the SHR-C group may be a reflection of the reductions in the enzymatic and nonenzymatic antioxidants defense system (31). Thus, the observations of the present study confirm those of a previous study that indicated that oxidative stress is present in hypertension (32). The results of the current study additionally illustrate that the oral administration of GSPE reduces the production of MDA.

SOD and CAT balance together to eliminate ROS, and small deviations in physiological concentrations may have marked effects on the resistance of cellular lipids, proteins and DNA to oxidative damage (33). Consistent with previous reports, SHRs indicated significantly depleted activities of the antioxidant enzymes SOD and CAT $(27,34)$. Upon GSPE treatment, antioxidant enzyme activities were significantly increased in the SHR-T group, and the high free radical scavenging activity of GSPE could be a possible reason for this reversal effect of the lipid peroxidation levels and antioxidative enzyme activities (35).

All of these results suggested that oxidative stress was closely involved in the development of arterial remodeling, and GSPE exerted a significantly beneficial effect on preventing the development of arterial remodeling by improving the antioxidant system, thus reducing oxidative stress.

\section{Acknowledgements}

The current study was supported by grants from the National Natural Science Foundation of China (grant no. 30700884), the Shandong Science and Technology Research Plan (grant no. 2010GGC10294), the Shandong Science and Technology Project Plan (grant no. 2012GB021817) and the National Science and Technology Major Project: Technology Platform Construction for Clinical Evaluation of Cardiovascular New Drug (grant no. 2012ZX09303016-003).

\section{References}

1. Chobanian AV, Bakris GL, Black HR, Cushman WC, Green LA, Izzo JL Jr, Jones DW, Materson BJ, Oparil S, Wright JT Jr, et al: Seventh report of the joint national committee on prevention, detection, evaluation, and treatment of high blood pressure. Hypertension 42: 1206-1252, 2003.

2. Behradmanesh S and Nasri P: Serum cholesterol and LDL-C in association with level of diastolic blood pressure in type 2 diabetic patients. J Renal Inj Prev 1: 23-26, 2012.
3. Intengan HD and Schiffrin EL: Vascular remodeling in hypertension: Roles of apoptosis, inflammation, and fibrosis. Hypertension 38: 581-587, 2001.

4. Tousoulis D, Briasoulis A, Papageorgiou N, Tsioufis C, Tsiamis E, Toutouzas K and Stefanadis C: Oxidative stress and endothelial function: Therapeutic interventions. Recent Pat Cardiovasc Drug Discov 6: 103-114, 2011.

5. Cakir Y and Ballinger SW: Reactive species-mediated regulation of cell signaling and the cell cycle: The role of MAPK. Antioxid Redox Signal 7: 726-740, 2005.

6. Lyle AN and Griendling KK: Modulation of vascular smooth muscle signaling by reactive oxygen species. Physiology (Bethesda) 21: 269-280, 2006.

7. McIntyre M, Bohr DF and Dominiczak AF: Endothelial function in hypertension: The role of superoxide anion. Hypertension 34: 539-545, 1999.

8. Berk BC: Redox signals that regulate the vascular response to injury. Thromb Haemost 82: 810-817, 1999.

9. Cave AC, Brewer AC, Narayanapanicker A, Ray R, Grieve DJ, Walker S and Shah AM: NADPH oxidases in cardiovascular health and disease. Antioxid Redox Signal 8: 691-728, 2006.

10. Bagchi D, Garg A, Krohn RL, Bagchi M, Bagchi DJ, Balmoori J and Stohs SJ: Protective effects of grape seed proanthocyanidins and selected antioxidants against TPA-induced hepatic and brain lipid peroxidation and DNA fragmentation, and peritoneal macrophage activation in mice. Gen Pharmacol 30: 771-776, 1998.

11. Liu X, Qiu J, Zhao S, You B, Ji X, Wang Y, Cui X, Wang Q and Gao H: Grape seed proanthocyanidin extract alleviates ouabain-induced vascular remodeling through regulation of endothelial function. Mol Med Rep 6: 949-954, 2012.

12. Li XL, Li BY, Gao HQ, Cheng M, Xu L, Li XH and Ma YB: Effects of grape seed proanthocyanidin extracts on aortic pulse wave velocity in streptozocin induced diabetic rats. Biosci Biotechnol Biochem 73: 1348-1354, 2009.

13. Guide for the Care and Use of Laboratory Animals. National Research Council (US) Institute for Laboratory Animal Research, National Academies Press (US), Washington (DC), 1996.

14. Rizzoni D, Paiardi S, Rodella L, Porteri E, De Ciuceis C, Rezzani R, Boari GE, Zani F, Miclini M, Tiberio GA, et al: Changes in extracellular matrix in subcutaneous small resistance arteries of patients with primary aldosteronism. J Clin Endocrinol Metab 91: 2638-2642, 2006.

15. Lopez-Andres N, Fortuno MA, Diez J, Zannad F, Lacolley P and Rossignol P: Vascular effects of cardiotrophin-1: A role in hypertension? J Hypertens 28: 1261-1272, 2010.

16. Wolinsky H: Long-term effects of hypertension on the rat aortic wall and their relation to concurrent aging changes. Morphological and chemical studies. Circ Res 30: 301-309, 1972.

17. Wang Y, Zhang J, Gao H, Zhao S, Ji X, Liu X, You B, Li X and Qiu J: Profilin-1 promotes the development of hypertension-induced artery remodeling. J Histochem Cytochem 62: 298-310, 2014.

18. Safar M, Chamiot-Clerc P, Dagher G and Renaud JF: Pulse pressure, endothelium function, and arterial stiffness in spontaneously hypertensive rats. Hypertension 38: 1416-1421, 2001.

19. Chan YH, Yiu KH, Lau KK, Yiu YF, Li SW, Lam TH, Lau CP, Siu CW and Tse HF: The CHADS2 and CHA2DS2-VASc scores predict adverse vascular function, ischemic stroke and cardiovascular death in high-risk patients without atrial fibrillation: Role of incorporating PR prolongation. Atherosclerosis 237: 504-513, 2014.

20. Tanaka M, Ishii H, Aoyama T, Takahashi H, Toriyama T, Kasuga H, Takeshita K, Yoshikawa D, Amano T and Murohara T: Ankle brachial pressure index but not brachial-ankle pulse wave velocity is a strong predictor of systemic atherosclerotic morbidity and mortality in patients on maintenance hemodialysis. Atherosclerosis 219: 643-647, 2011.

21. Ng K, Butlin M and Avolio AP: Persistent effect of early, brief angiotensin-converting enzyme inhibition on segmental pressure dependency of aortic stiffness in spontaneously hypertensive rats. J Hypertens 30: 1782-1790, 2012.

22. Rudic RD and Sessa WC: Nitric oxide in endothelial dysfunction and vascular remodeling: Clinical correlates and experimental links. Am J Hum Genet 64: 673-677, 1999.

23. Fujita K, Matsumura Y, Kita S, Miyazaki Y, Hisaki K, Takaoka M and Morimoto S: Role of endothelin-1 and the ETA receptor in the maintenance of deoxycorticosterone acetate-salt-induced hypertension. Br J Pharmacol 114: 925-930, 1995. 
24. Statsenko ME and Derevianchenko MV: Correction of endothelial dysfunction in hypertensive patients with type II diabetes mellitus during combined antihypertensive therapy. Ter Arkh 86: 90-93, 2014 (In Russian).

25. Zhu WW, Liu XP, Wu N, Zhao TT, Zhao Y, Zhang J and Shao JH: Beneficial effects of losartan on vascular injury induced by advanced glycosylation end products and their receptors in spontaneous hypertension rats. Mol Cell Biochem 304: 35-43, 2007.

26. Jalili T, Carlstrom J, Kim S, Freeman D, Jin H, Wu TC, Litwin SE and David Symons J: Quercetin-supplemented diets lower blood pressure and attenuate cardiac hypertrophy in rats with aortic constriction. J Cardiovasc Pharmacol 47: 531-541, 2006.

27. Boghdady NA: Antioxidant and antiapoptotic effects of proanthocyanidin and ginkgo biloba extract against doxorubicin-induced cardiac injury in rats. Cell Biochem Funct 31: 344-351, 2013.

28. Ozkan G, Ulusoy S, Alkanat M, Orem A, Akcan B, Ersöz S, Yuluğ E, Kaynar K and Al S: Antiapoptotic and antioxidant effects of GSPE in preventing cyclosporine A-induced cardiotoxicity. Ren Fail 34: 460-466, 2012.
29. Shao ZH, Wojcik KR, Dossumbekova A, Hsu C, Mehendale SR, Li CQ, Qin Y, Sharp WW, Chang WT, Hamann KJ, et al: Grape seed proanthocyanidins protect cardiomyocytes from ischemia and reperfusion injury via Akt-NOS signaling. J Cell Biochem 107: 697-705, 2009.

30. Belcaro G,Ledda A,Hu S, Cesarone MR, Feragalli B and Dugall M: Grape seed procyanidins in pre- and mild hypertension: A registry study. Evid Based Complement Alternat Med 2013: 313142, 2013.

31. Yu BP: Cellular defenses against damage from reactive oxygen species. Physiol Rev 74: 139-162, 1994.

32. González J, Valls N, Brito R and Rodrigo R: Essential hypertension and oxidative stress: New insights. World J Cardiol 6: 353-366, 2014.

33. Matés JM and Sánchez-Jiménez F: Antioxidant enzymes and their implications in pathophysiologic processes. Front Biosci 4: D339-D345, 1999.

34. Zheng $\mathrm{H}$ and $\mathrm{Yu}$ YS: Chronic hydrogen-rich saline treatment attenuates vascular dysfunction in spontaneous hypertensive rats. Biochem Pharmacol 83: 1269-1277, 2012.

35. Ulker S, McMaster D, McKeown PP and Bayraktutan U: Impaired activities of antioxidant enzymes elicit endothelial dysfunction in spontaneous hypertensive rats despite enhanced vascular nitric oxide generation. Cardiovasc Res 59: 488-500, 2003. 